\title{
Overview of Routing Protocols in VANET
}

\author{
Dhyey Patel \\ Student Thadomal \\ Shahni Engineering \\ College, Mumbai
}

\author{
Mohd. Faisal \\ Khan \\ Student Thadomal \\ Shahni Engineering \\ College, Mumbai
}

\author{
Priyanka \\ Batavia \\ Student Thadomal \\ Shahni Engineering \\ College, Mumbai
}

\author{
Sidharth \\ Makhija \\ Student Thadomal \\ Shahni Engineering \\ College, Mumbai
}

\author{
M. Mani Roja \\ Asso. Professor \\ Thadomal Shahni \\ Engineering \\ College, Mumbai
}

\begin{abstract}
Vehicular Ad-hoc Network (VANET) is an upcoming and emerging technology for intelligent transport technology (ITS) that enables Vehicle to Vehicle $(\mathrm{V} 2 \mathrm{~V})$ and Vehicle to Infrastructure (V2I) communication by using various IEEE standards such as IEEE 802.11p. The high mobility of nodes in VANETs creates major challenges in routing the information packets to desired destination node which need to be addressed by differently devised routing protocols. Taking this into consideration, the paper gives an overview and tabular comparison of VANET and its various routing protocols with the prime focus given to protocols of V2V communication.
\end{abstract}

\section{Keywords}

VANET, communication, protocols, routing, V2V.

\section{INTRODUCTION}

Vehicular accidents kill millions of people across the globe. Safety is a dominating factor in designing many vehicular automation systems. Major attention is given to minimizing the probability of failure occurring and improvising the reliability of all elements of the system. A mobile ad hoc network (MANET) is a network that has many free or autonomous nodes, composed of mobile devices that can change locations and is a self-configuring, infrastructure-less network of wireless mobile devices that can be connected to Wi-Fi or any cellular system. Vehicular Ad Hoc Network (VANET) is a wireless ad hoc network, which uses moving vehicles as nodes and allows them to connect with each other via wireless network with characteristics such as predictable mobility, rapid changing topology, high computational ability and variable network density. Infrastructure-to-vehicle (I2V), vehicle-to-infrastructure (V2I), and vehicle-to-vehicle (V2V) communication systems are enabled by vanet. Vehicle-toInfrastructure (V2I) Communications is a wireless exchange of safety and operational data between vehicles and roadway infrastructure, intended majorly to avoid motor vehicle collisions. V2V system is a type of network in which nearby vehicles use dynamic wireless exchange of data, providing each other with relative proximity information. When two or more vehicles are in radio communication range, it results in automatic connection and establishment of ad hoc network which enables sharing of position, speed, and direction data. Dedicated short range communication (DSRC) combines with GPS resulting in V2V communication system which provides a 360-degree view of vehicles within the communication range. Latency-sensitive collision and Collision between vehicles out of line of sight can be avoided by VANET using DSRC and GPS [1].

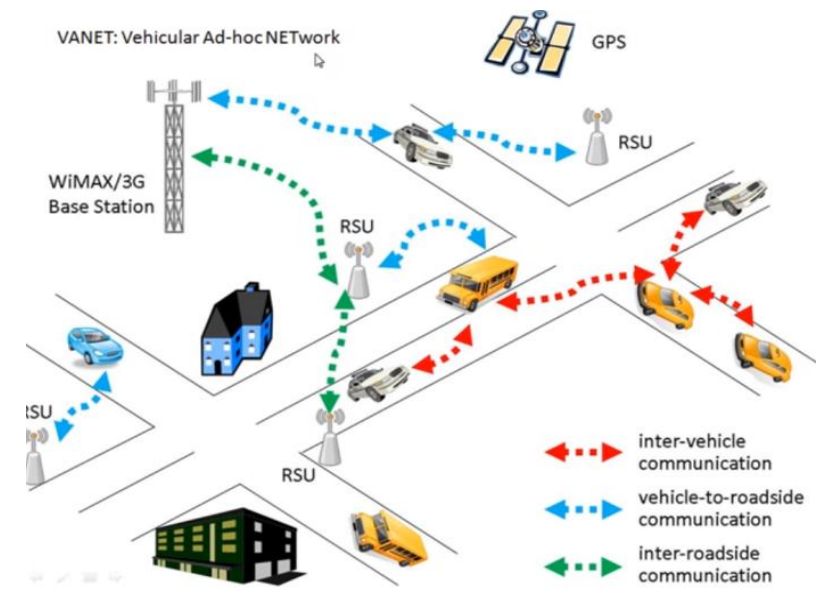

Fig 1: VANET Communication [2]

\section{ROUTING PROTOCOLS IN VANET}

The main purpose of routing protocols is to obtain minimal communication time while using minimum amount of network resources [3]. The five categories of routing protocols are: Topology based routing, Position based routing, Geo cast routing, Broadcast routing and Cluster based routing. These are classified based on the region of application where they are most suitable.

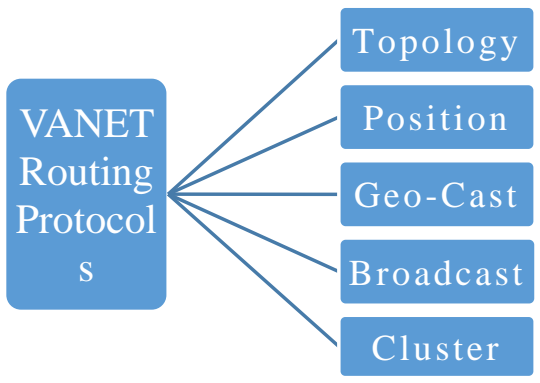

Fig 2: Flowchart of routing protocols in VANET.

\subsection{Topology Routing}

The link's information within the network is used to send the data packets from source to destination in topology based routing protocol. Packet forwarding is performed by using link's information existing in the present network. Topology based routing protocols are categorized into proactive (tabledriven) and reactive (on-demand) routing $[4,5]$.

\subsubsection{Proactive protocol}

Each node continuously maintains routing information forwarded in the next hop which routes to every other node in the network background irrespective of communication request [3]. Since the route from source to destination is stored at all times, this protocol has low latency. Now as the destination route is stored in the background there is no need 
for route discovery and hence is the advantage. Wireless Routing Protocol (WRP) and Global State Routing (GSR) are some of the examples of proactive protocols $[4,5]$.

\subsubsection{Ad hoc/Reactive routing protocol}

Reactive routing opens the route only when it wants to send packets to its destination for a node to communicate with each other. It maintains only the routes that are currently in use till the destination becomes inaccessible along every path from the source as a result it reduces the burden of the network. Cluster Based Routing Protocol (CBRP), Signal Stability Routing (SSR) and Location Aided Routing (LAR) are some types of reactive protocols $[4,6]$.

\subsection{Position Routing}

Position based routing protocol uses location services to determine the exact position of source node, neighbor node and the destination node. By the use of GPS, it maintains the position information about the nodes and determines the exact co-ordinates of the nodes in all directions, resulting in route discovery mechanism. When the source node is ready to transmit a packet, it should get the location co-ordinates $(\mathrm{x}, \mathrm{y})$ of the destination [7]. Neither it requires the establishment and maintenance of the route, nor does it have to update the routing table. It does all its activity by the use of location tracking and some kind of forwarding strategy, which is implemented in forwarding the packets via the source node [4]. The advantages of Position based routing are:

- $\quad$ Route discovery \& management is not required.

- Scalability

- Compatible with high node mobility.

A shortcoming of Position based routing is the need for position determining services. Two examples of Position based routing are:

- Position Based Greedy V2V Protocols.

- Delay Tolerant Protocols.

\subsubsection{Position Based Greedy V2V Protocols}

In greedy strategy an intermediate node in the route transmits the message to the farthest neighbor towards the next destination. Greedy approach requires that intermediate node should possess the position of itself, position of its neighbor and destination position and the next hop is decided depending on the optimization criteria of the algorithm. Most position-based protocols use greedy forwarding to route packets from a source to the destination. This protocol transmits data packets to its destination as soon as possible, therefore these protocols are also known as minimum delay routing protocols [5].

\subsubsection{Delay Tolerant Protocols}

In a densely packed urban scenario, locating a node to carry a message is not a problem but in reduced traffic situations such as rural highways, establishing end to end route is difficult. In such cases certain consideration needs to be given in sparse networks. The various types of Delay Tolerant Protocols are MOVE, VADD, and SADV [5, 9].

\subsection{Geo-cast Routing}

Geo-cast routing is a location based multicast routing with the primary objective to deliver the packet from source node to other nodes within a specified geographical region (Zone of Relevance ZOR). The vehicles outside the ZOR are not alerted by geo-casting routing to avoid unnecessary hasty reaction. Geo-cast has a specific geographic region which defines a forwarding zone where the flooding of packets is directed in order to reduce network congestion and message overhead caused by simply flooding packets everywhere. The various Geo-cast routing protocols are IVG, ROVER, DGCASTOR and DRG [5]. Its disadvantage is high latency period and contention \& collision problems. Reduction in overhead transmission of data packets is one of the advantages.

\subsubsection{Inter-Vehicle Geo-cast (IVG)}

IVG is a Geo-cast routing protocol which is for disseminating safety alert messages to vehicles on highways. Timer based mechanism is used for forwarding message. For overcoming network fragmentation periodic broadcasts is used [5].

\subsubsection{Robust Vehicular Routing (ROVER)}

Robust Vehicular Routing [4] is a Geo-cast based Routing Protocol which broadcasts messages to other vehicles within a specified Zone of Relevance (ZOR) [9]. In this protocol control packets are broadcasted in the network and the data packets are unicasted. It functions as reliable geographical multicast protocol. The drawbacks of this protocol are delay in data transfer because of redundant messages and control packet overhead is high. Dynamic Time-Stable Geo-Cast Routing (DTSG) Dynamic Time-Stable Geo-Cast Routing [9] is designed for sparse density networks. This protocol has a pre-stable phase which helps the message to be disseminated within the region and stable period intermediate node which uses store and forward method for a predefined time within the region. It dynamically adjusts network density and the vehicles speed for better performance.

\subsection{Broadcast Routing}

In broadcast protocol, the brake light and the left/right turn indicator lights are replaced by the communication devices will deliver signaling messages to other vehicles such as audio or image $[6,11]$. Since the warning message is received, the driver will drive the vehicle safely. The reason for analyzing broadcasting protocols is traffic information, weather and emergency information, road conditions among vehicles and delivering advertisements and announcements. Broadcast routing protocol is flooding based routing protocol which is used in VANET for sharing information among vehicles such as when an accident or an event occurs then transmit the information to maximum nodes possible. In most of the emergency situations the messages to be delivered have to be quick and efficient, hence broadcast based routing protocols plays a vital role in almost all safety applications. With the help of multi-hop transmission the information is distributed to the vehicles beyond the transmission range using broadcast protocol. Conventional ad-hoc routing protocols such as DSR and AODV [3] will not be appropriate in VANETs for most vehicular broadcast applications. The major disadvantages in the broadcast routing protocol are the hidden node problem and the high possibility of collision in the messages. Messages to vehicles within the communication range are delivered by broadcasting protocols and it relays the messages to all the vehicles in the network. Examples of broadcasting protocols are BROADCOMM and DV-CAST [4, 5].

\subsubsection{Distributed Vehicular Broadcast Protocol}

DV-CAST $[8,11,12]$ is based on the level of connectivity of the nodes such as well connected, sparsely connected, and totally disconnected neighborhood that divides vehicles into three categories. In a well-connected neighborhood persistence scheme is used, for sparsely connected 
neighborhood vehicles rebroadcast to the vehicles moving in the same direction after receiving the broadcast message and for totally disconnected neighborhood vehicles store the broadcast message until another vehicle enters into transmission range. A redundant packet is detected using a flag variable $[8,11]$.

\subsubsection{BROADCOMM}

It is based on hierarchal structure, which is used for highway network. In this protocol highway is segmented to virtual cells, which move along with the vehicles. There are two level of hierarchy for the nodes in the highway. All the nodes in a cell are included in first level. The second level is represented by cell reflectors, which are responsible for handling messages within its cell nodes and forwarding or receiving the messages to or from neighboring cell reflectors. This protocol is suited for simple highway structure, which contains smaller number of nodes. However, the position information is fully dependent on formation of cells [6].

\subsection{Cluster Routing}

Cluster based routing protocol establishes a cluster between nodes/vehicles. A group of nodes are classified as a cluster and each cluster has a single unique cluster-head, which is responsible for intra and inter-cluster communication. In order to communicate within the cluster a direct link is setup between the nodes and inter-cluster communication is only possible via the designated cluster-heads. In cluster based routing, the cluster-head broadcasts the packet to all the nodes in the cluster, this results in improved scalability for large a network of nodes although the network delay and overhead increase for high mobility feature of VANET. The various Clusters based routing protocols are HCB, CBDRP, CBLR and CBR [4, 5, and 9].
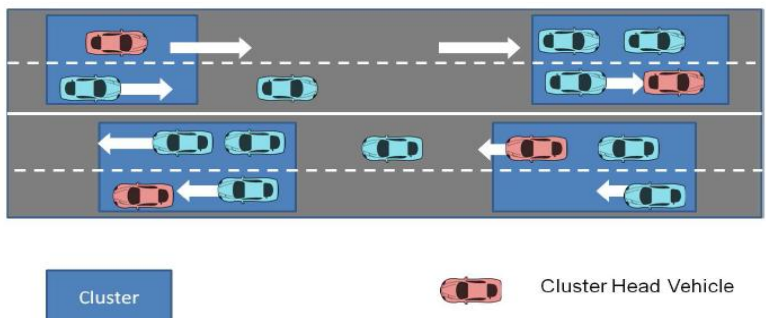

Cluster Head Vehicle

Fig 3: Cluster Based Routing [6]

\subsubsection{Cluster-Based Directional Routing Protocol (CBDRP)}

CBDRP is designed for vehicles, which are moving in same direction. It is the responsibility of the cluster-head to forward the packet within the cluster when it receives a packet from another cluster-head. This protocols behavior is like CBR but direction and velocity are considered when packet forwarding. Its advantages are rapid and reliable data transfer and improved stability. A few issues with this protocol are that the control packet overhead is average and the number of retransmission is high [5].

\subsubsection{Cluster Based Location Routing (CBLR)}

CBLR $[5,6]$ is not only clustering based protocol but also a reactive protocol. Addresses and locations of the cluster members are contained in the routing table which is used by each cluster header. The mechanism for packet transmission is that when a source node wants to send a packet to the destination then it sends the packet to the neighbor closest to the destination if it is in same cluster. If destination is in another cluster, then it stores the data packet in its buffer then broadcasts Location Request (LREQ) [5] packets and starts a timer. Its pros are that it is suitable for high mobility networks and control packet overhead is low [5]

\subsubsection{Location Routing Algorithm with Cluster- Based Flooding (LORA-CBF):}

LORA-CBF is similar to greedy routing. The cluster-head maintains the information between nodes. When two clusters are connected by a node then it is called gateway. When destination of a node is not available then cluster-head and gateways send location request (LREQ) packets [5]

\section{COMPARATIVE REVIEW}

The routing protocols have been evaluated using a tabular comparison. As seen in Table 1 the routing protocols best suited for the urban environment are Position and Cluster based protocols owing to their ability to handle large number of nodes. Geo-cast and Broadcast protocols are better suited for scenarios wherein the number of nodes are less and mobility of nodes is high, though the network overhead for Broadcast based routing is an issue for current VANET systems.

Table 1: Comparison of Routing Protocols in VANET [5]

\begin{tabular}{|c|c|c|c|c|c|}
\hline $\begin{array}{c}\text { Types of } \\
\text { protocols }\end{array}$ & $\begin{array}{c}\text { Topolo } \\
\text { gy } \\
\text { based }\end{array}$ & $\begin{array}{c}\text { Positi } \\
\text { on } \\
\text { based }\end{array}$ & $\begin{array}{c}\text { Cluste } \\
\text { r } \\
\text { based }\end{array}$ & $\begin{array}{c}\text { Geo- } \\
\text { Cast } \\
\text { based }\end{array}$ & $\begin{array}{c}\text { Broad } \\
\text { cast } \\
\text { based }\end{array}$ \\
\hline $\begin{array}{c}\text { Forwardi } \\
\text { ng } \\
\text { technique }\end{array}$ & $\begin{array}{c}\text { Wirele } \\
\text { ss } \\
\text { multi } \\
\text { hop } \\
\text { forwar } \\
\text { ding }\end{array}$ & $\begin{array}{c}\text { Heuris } \\
\text { tic } \\
\text { Techni } \\
\text { que }\end{array}$ & $\begin{array}{c}\text { Wirele } \\
\text { ss } \\
\text { multi } \\
\text { hop } \\
\text { forwar } \\
\text { ding }\end{array}$ & $\begin{array}{c}\text { Wirele } \\
\text { ss } \\
\text { multi } \\
\text { hop } \\
\text { forwar } \\
\text { ding }\end{array}$ & $\begin{array}{c}\text { Wirele } \\
\text { ss } \\
\text { multi } \\
\text { hop } \\
\text { forwar } \\
\text { ding }\end{array}$ \\
\hline $\begin{array}{c}\text { Strategy } \\
\text { of } \\
\text { recovery }\end{array}$ & $\begin{array}{c}\text { Multi } \\
\text { Hop } \\
\text { forwar } \\
\text { ding }\end{array}$ & $\begin{array}{c}\text { Carry } \\
\text { Forwa } \\
\text { rd } \\
\text { approa } \\
\text { ch }\end{array}$ & $\begin{array}{c}\text { Carry } \\
\text { Forwar } \\
\text { d } \\
\text { approa } \\
\text { ch }\end{array}$ & $\begin{array}{c}\text { Floodi } \\
\text { ng }\end{array}$ & $\begin{array}{c}\text { Carry } \\
\& \\
\text { Forwar } \\
\text { d } \\
\text { approa } \\
\text { ch }\end{array}$ \\
\hline $\begin{array}{c}\text { Digital } \\
\text { Map } \\
\text { requirem } \\
\text { ent }\end{array}$ & No & No & Yes & No & No \\
\hline $\begin{array}{c}\text { Virtual } \\
\text { Infrastru } \\
\text { cture } \\
\text { requirem } \\
\text { ent }\end{array}$ & No & No & Yes & No & No \\
\hline $\begin{array}{c}\text { Realistic } \\
\text { Traffic } \\
\text { Flow }\end{array}$ & Yes & Yes & No & Yes & Yes \\
\hline $\begin{array}{c}\text { Scenario } \\
\text { Urban }\end{array}$ & $\begin{array}{c}\text { Urban } \\
\text { Urban }\end{array}$ & $\begin{array}{c}\text { Highw } \\
\text { ay }\end{array}$ & $\begin{array}{c}\text { Highw } \\
\text { ay }\end{array}$ \\
\hline & & & & \\
\hline
\end{tabular}

\section{CONCLUSION}

VANET is an important emerging field, source of research and area of development which results in advanced intra vehicular communication with the help of advancements in mobile communication. In this paper by comparing and evaluating the different routing protocols it is observed that the designing of VANET protocols is challenging owing to extreme mobility of the vehicles. Since one of the main 
advantages of using VANET systems is avoiding collisions between vehicles by reducing human error, having a secure connection between nodes also becomes essential. In the near future, research and development of efficient routing protocols in VANETs will be at the heart of collision avoidance systems and will play a pivotal role in the development of automated vehicles. The routing protocols need to be designed considering factors such as the security, mobility and scalability of vehicular communication.

\section{REFERENCES}

[1] "Inter-Vehicular Communication Systems", Daniel López García, (lgdaniel@hotmail.com) Danckelmannstrasse 46/47 Berlin, Germany. Available: http://www.mi.fu-berlin.de/inf/groups/agtech/teaching/2011_SS/S_19510b_Proseminar_Technisc he_Informatik/daniel-lopez-report.pdf

[2] "Research Issues on Vehicular Ad hoc Network", International Journal of Engineering Trends and Technology (IJETT) - Volume 6 Number 4- Dec 2013

[3] P. Tomer, M. Chandra, "An application of routing protocols for Vehicular Ad-hoc Networks ", Networking and Information Technology (ICNIT), 2010 IEEE International Conference on 11-12 June 2010.

[4] "A Comparative Study of Various Routing Protocols in VANET", IJCSI International Journal of Computer Science Issues, Vol. 8, Issue 4, No 1, July 2011. http://arxiv.org/ftp/arxiv/papers/1108/1108.2094.pdf
[5] "Survey over VANET Routing Protocols for Vehicle to Vehicle Communication", IOSR Journal of Computer Engineering (IOSRJCE) ISSN: 2278-0661, ISBN: 22788727 Volume 7, Issue 5 (Nov-Dec. 2012), PP 01-09

[6] "A Survey of Vehicular Ad hoc Networks Routing Protocols", International Journal of Innovation and Applied Studies ISSN 2028-9324 Vol. 3, No. 3, July 2013, pp. 829-846

[7] "A Survey on Position-based routing", International Journal of P2P Network Trends and Technology (IJPTT) -Volume3 Issue7-August 2013

[8] "Fuel Consumption and throughput Optimization in Vanet", International Journal of Latest Trends in Engineering and Technology (IJLTET), Vol. 5 Issue 3 May 2015, ISSN: 2278-621X.

[9] "Study of Efficient Routing Protocols for VANET", International Journal of Scientific \& Engineering Research Volume 4, Issue3, March-2013, ISSN 22295518.

[10] M.Kihl, "Reliable Geographical Multicast Routing in Vehicular Adhoc Networks", 2007.

[11] "Vehicular Ad-Hoc Networks (VANETs) - An Overview and Challenges" Journal of Wireless Networking and Communications, 2013, p-ISSN: $2167-$ 7328 e-ISSN: 2167-7336.

[12] "Routing Protocols in Vehicular Ad Hoc Networks: A Survey and Future Perspectives", JISE-2010. 